\title{
Participation to the first Cybathlon: an overview of the FREEWHEELS team FES-cycling solution
}

Benoît Sijobert (1), Charles Fattal (2), Anne Daubigney (3), Christine Azevedo-Coste (1)

(1) INRIA - LIRMM Université de Montpellier, Montpellier, France; (2) CRF La Chataîgneraie, Menucourt, France; (3) COS Divio, Dijon, France

This article is distributed under the terms of the Creative Commons Attribution Noncommercial License (CC BY-NC 4.0) which permits any noncommercial use, distribution, and reproduction in any medium, provided the original author(s) and source are credited.

\begin{abstract}
This article is a contribution to a special issue aiming at collecting data and documenting the different specificities of the teams which participated into Cybathlon 2016 FES-bike discipline. Our team prepared one paraplegic pilot over one year and developed a FES-cycling device based on existing commercial products. Our pilot (47 y.o, spinal cord lesion T3 AIS A since year 1995) was qualified for the final race and finished in 6th position over 12 participants in the discipline, covering a total distance of $750 \mathrm{~m}$ at an average speed of $5.71 \mathrm{~km} / \mathrm{h}$, propelled by his own quadriceps and hamstrings muscles.
\end{abstract}

Key Words: FES cycling, spinal cord injuries, Cybathlon

Eur J Transl Myol 27 (4): 265-271

In 2016 an academic research team (CAMIN, INRIA, Montpellier, France) and a rehabilitation center team (COS DIVIO, Dijon, France) have participated into Cybathlon competition, in the FES-bike discipline under the name of FreeWheels team. ${ }^{1}$ Despite more than 14 years investigating on Functional Electrical Stimulation (FES) applications and more broadly in the field of Neuroprostheses, ${ }^{2-6}$ using FES for cycling was a first for the research team. The FES-bike discipline consisted of a series of races between two spinal cord injured (SCI) participants propelling a cycling device by means of their lower limbs muscular contractions elicited by FES. The participants had to cover a 750 meters distance in less than 8 minutes, starting from a $10^{\circ}$ descending ramp. Mobile and stationary cycling using FES have been widely investigated since the 1980s through many studies. $^{7-13}$ It has been shown that FES-cycling of subjects with SCI results in physiological and psychological positive effects such as cardiovascular training, decrease in pressure sores occurrence and selfesteem improvements. ${ }^{14-16}$ However, the use of this technology has often remained restricted to indoor and stationary ergometers in clinical contexts, partly due to the small amount $(10-25 \mathrm{~W})$ of power produced, ${ }^{17,18}$ the requirement of experimented users and the lack of commonly available affordable FES outdoor bikes. Cybathlon FES-bike challenge motivated considerable efforts in technical developments to allow the coverage of a significant distance at a suitable speed.

This event promoted the activity as recreational with potential mobility applications. In the following we describe the different characteristics of our pilot and device.

\section{Material and Methods}

This study was conducted in accordance with the principles of the Declaration of Helsinki and approved by the local Ethics Committee (CPP Dijon, ID RCB: 2016-A00279-42), including the participant selection, physical preparation, training and participation into Cybathlon. One subject was included, the so-called pilot.

\section{Inclusion and exclusion criteria}

The Cybathlon competition rules constrained the participant to present an ASIA A or B spinal cordinjury, with a complete loss of motor function. We have furthermore defined the following inclusion criteria: age $\geq 18$ years and $<65$ years, complete traumatic lesion $>$ 12 months, neurological level T2 to T12, stable medical status, stimulable sublesional muscles and passive joint movement in the lower limbs. Exclusion criteria were defined as follows: body mass index $\geq 30$, pressure ulcer, thrombophlebitis, neurogenic paraosteoarthropathy, cardiovascular disease, muscle disease, DXA T-score <2.5, hip or knee arthroplasty, epilepsy, hypotension, lower limb fracture within the past 12 months, a pacemaker or other implant, and pregnancy. No betablocker treatment was authorized during the experiment.

\section{Pilot profile}

The pilot was selected in respect with the inclusion criteria listed in the previous paragraph. He was 47 years 


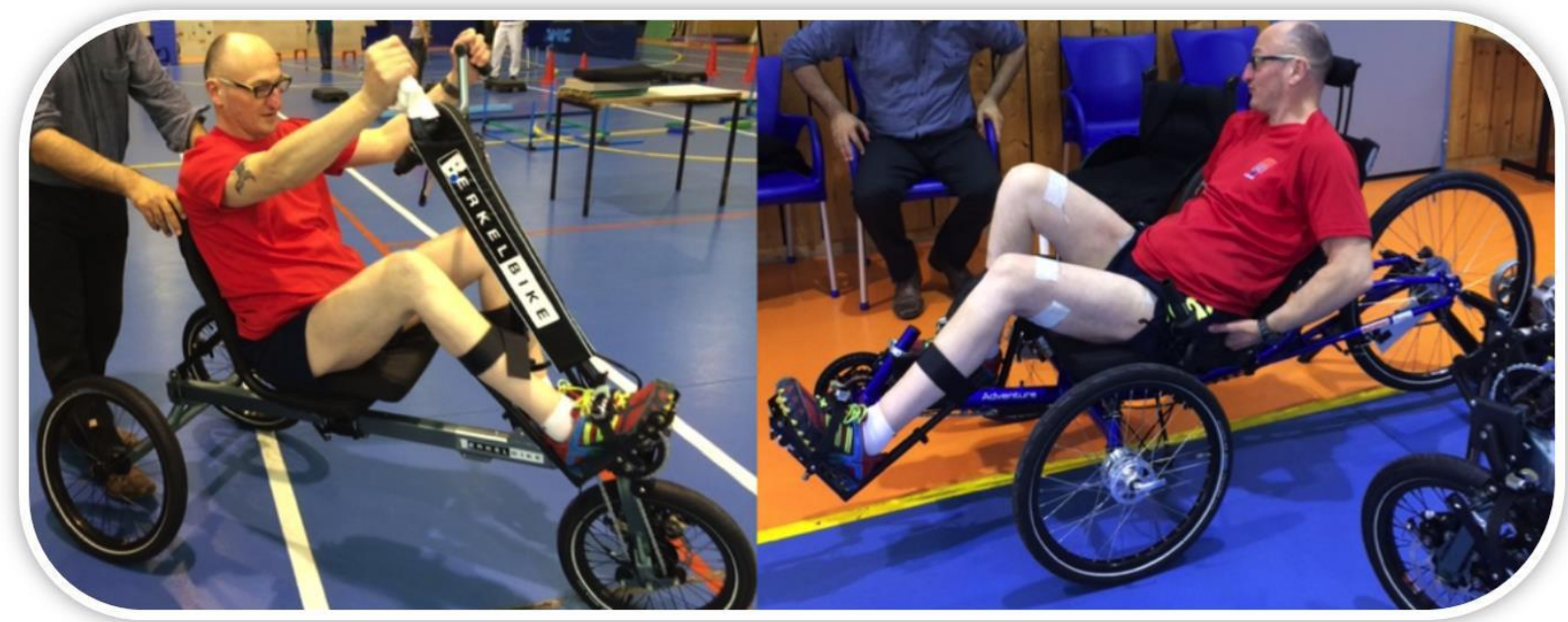

Fig 1. The two trikes used (left) during the initial training program, the Berkelbike Pro® and (right) for the final training program and at the competition, the FreeWheels trike

old $(1 \mathrm{~m} 78,74 \mathrm{~kg})$ at the time of inclusion and presented a spinal cord lesion T3 (AIS A) since 1995.

\section{Tricycle description}

Two distinct cycling devices were used during the study (Fig. 1).

A the Berkelbike Pro®, a commercial tricycle designed for persons with physical limitations.

B the FreeWheels trike, an instrumented recumbent cycle adapted from a commercial device, the ICE
Trike Adventure ${ }^{\circledR}$ designed for non disabled individuals.

The Berkelbike Pro® was chosen to allow the pilot to train before the FreeWheels competition device was finalized (3 months before the competition). No modification was done on the device. The choice of the competition tricycle was based on several considerations. The first requirement concerned accessibility allowing for safe wheelchair/trike transfers (retractable handles,

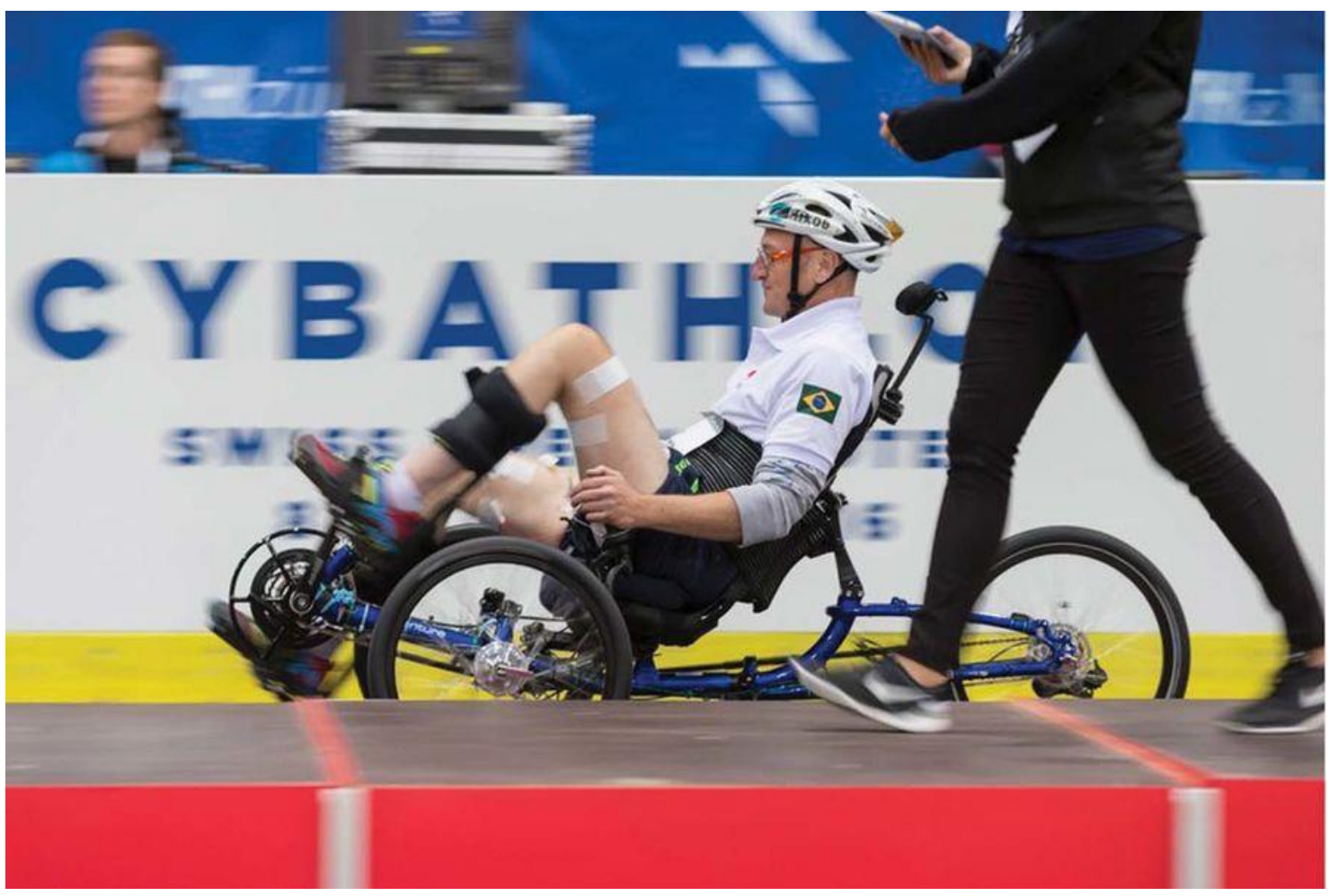

Fig 2. FreeWheels pilot during the Cybathlon final race. Photos credits : geschützt 

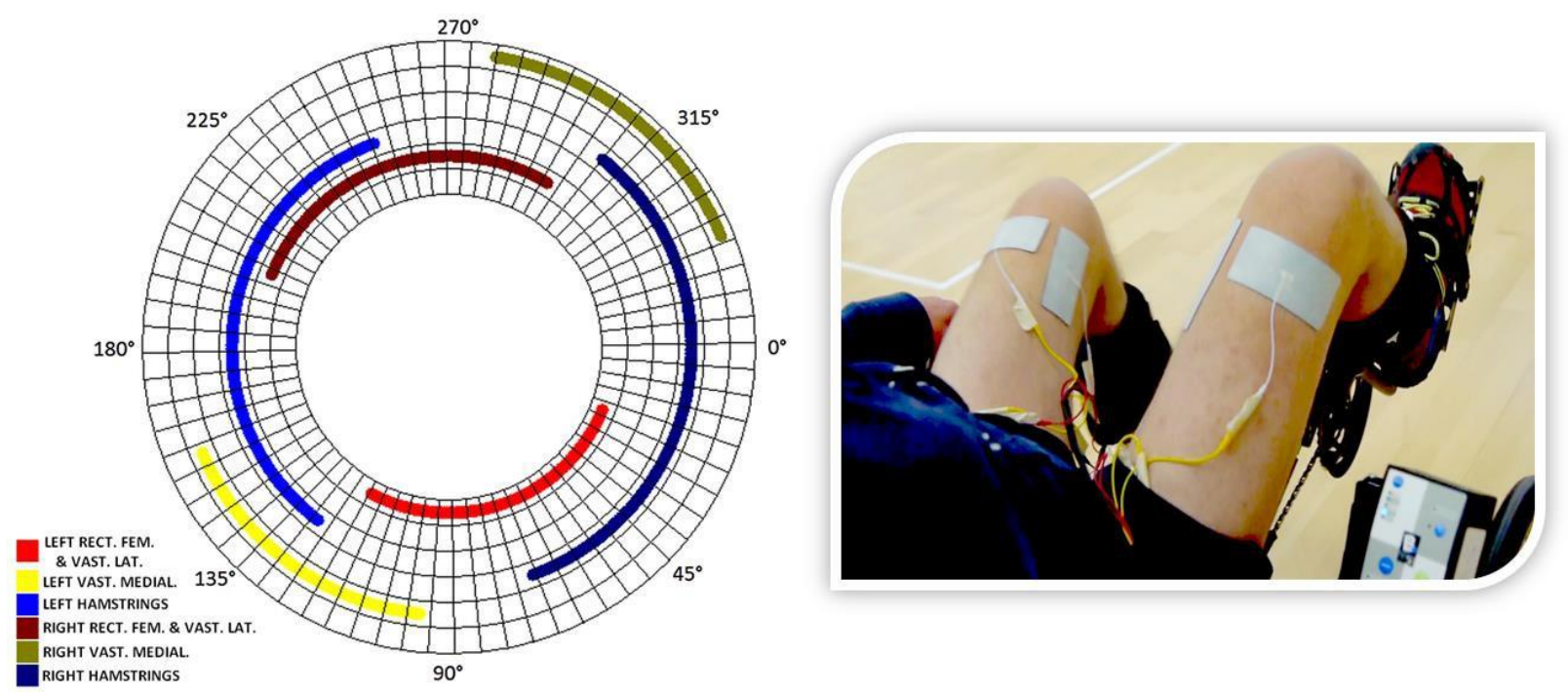

Fig 3. Stimulation pattern designed with individualized quadriceps and hamstrings. On each leg, one channel is dedicated to the rectus femori/vastus lateralis muscles, a second channel to the vastus medialis and a third one to the hamstrings

low height, high stability, etc ...). In order to later facilitate diffusion of the approach we also wanted the device to be affordable, foldable and sufficiently light for transportation. We have selected the ICE Adventure 26
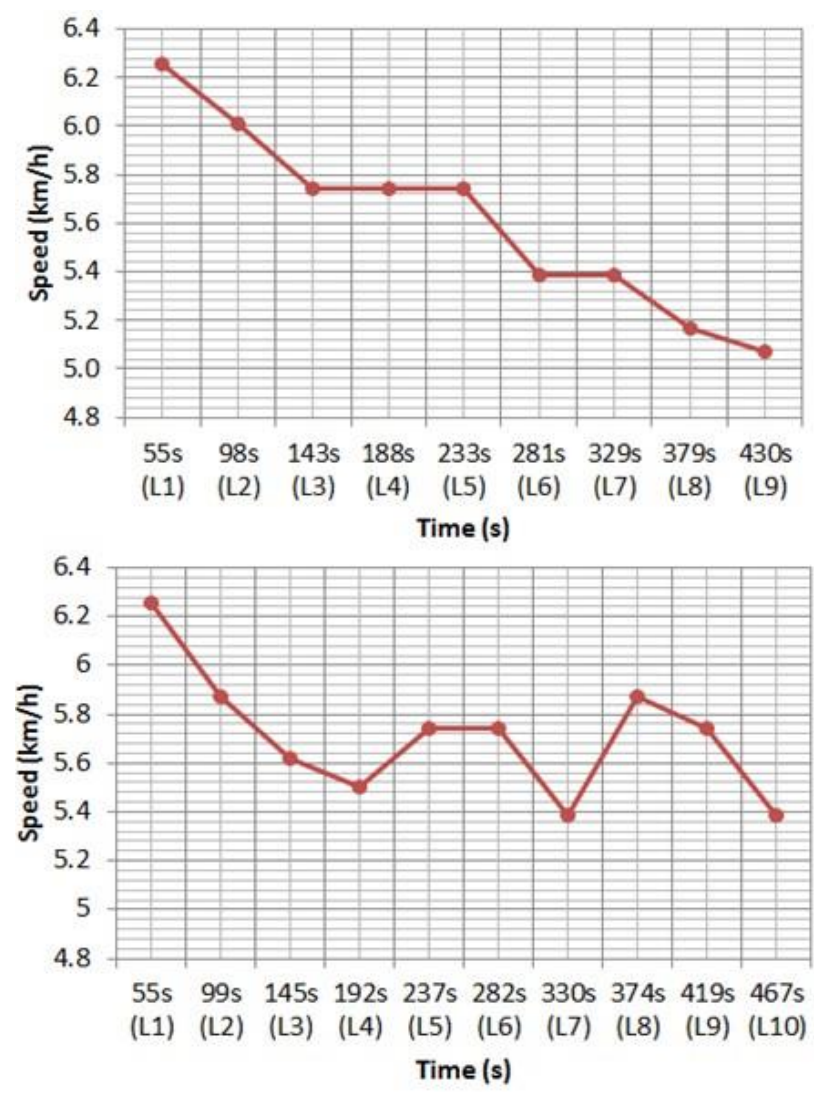

Fig 4. Average speed $(\mathrm{km} / \mathrm{h})$ computed on each lap (from L1 to L10) during the Cybathlon qualification (top) and final (bottom) races model (cost $€ 3000$, foldable steel frame, overall weight of $17.5 \mathrm{Kg}$ and $2 \mathrm{~m}$ length). This product has a mesh seat, designed to provide an optimum back support and comfort. The tension in the back could be adjusted. The cover features is made on a breathable fabrics to maximise airflow. A VICAIR®-type pressure relief cushion was chosen by the team occupational and physiotherapists and then fixed on the seat to avoid sliding. Hase ${ }^{\circledR}$ pedals with calf support were adapted to ensure a sufficient lateral locking of the legs while holding the ankle joint at $90^{\circ}$. The ICE trike was initially equipped with a $26^{\prime}$ rear wheel, we changed for a 24 " one to tilt the bike and elevate the crank relatively to the pilot's position (Fig. 2). Low rolling resistance tires (Schwalbe ${ }^{\circledR}$ Kojak 24 x 1.35") were also mounted instead of the originaltires.

A few weeks before Cybathlon competition we decided

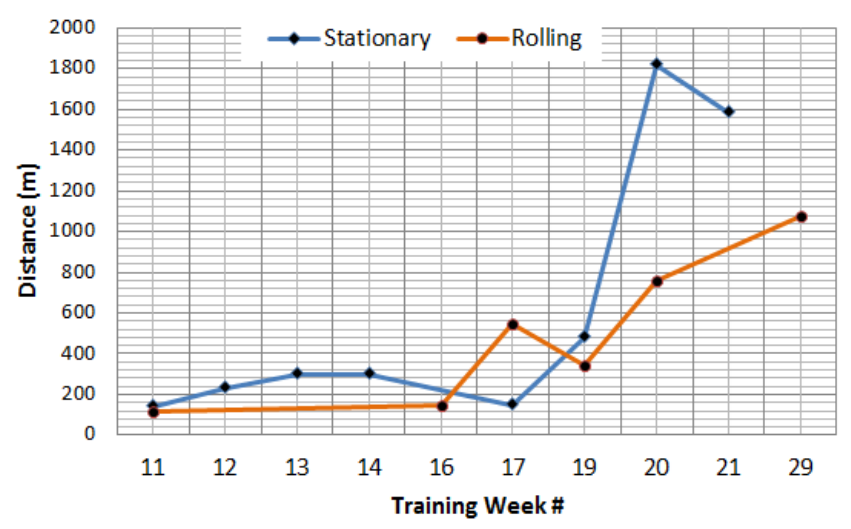

Fig 5. Maximum covered distance during the training sessions, from M9 to M13 (from week 11th to week 29th of the FES-cycling program) 

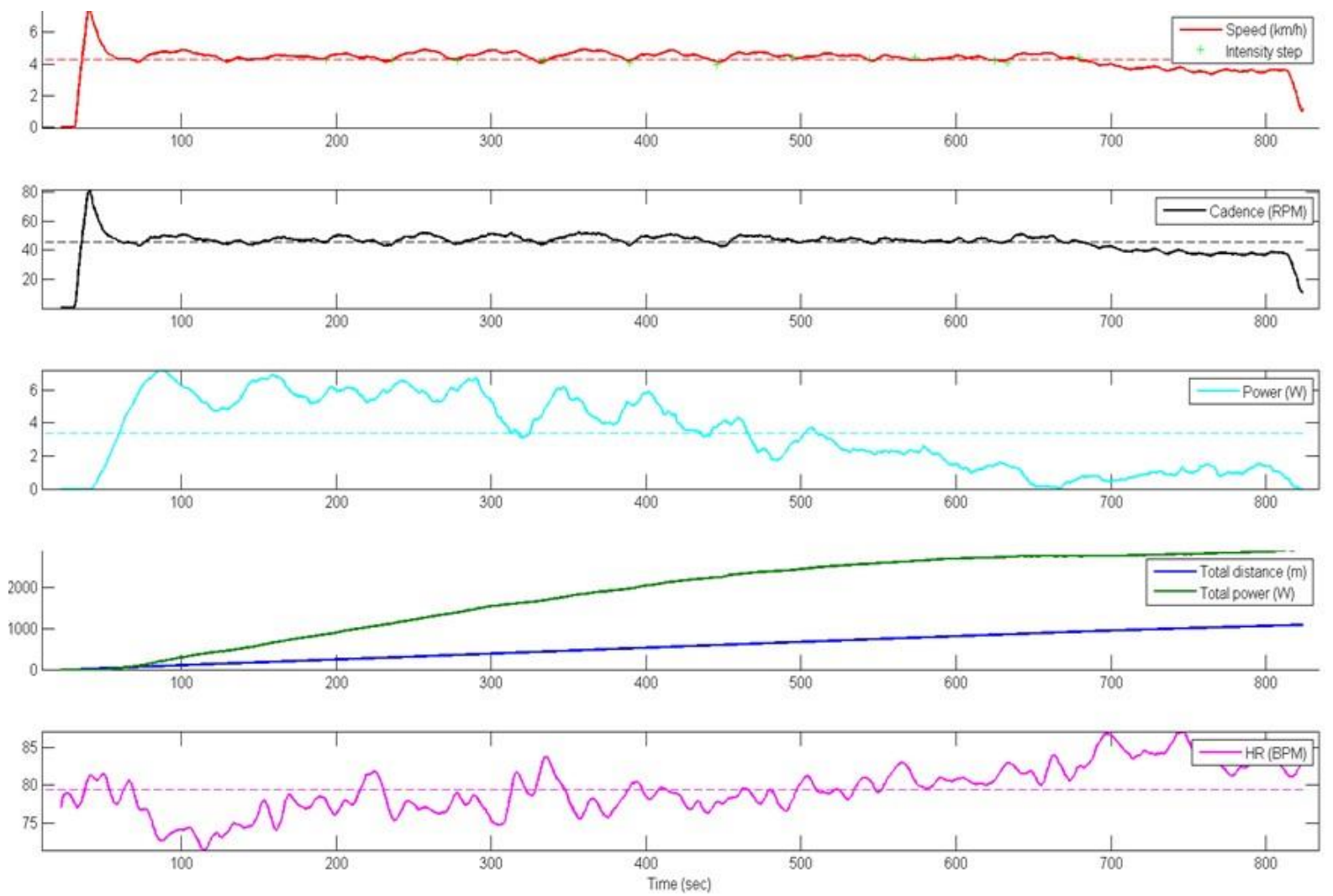

Fig 6. Data overview collected during a final evaluation race at M13.

to modify the rear wheel transmission, initially a freewheel, to a fixed-gear. This decision was made in consequences of difficulties to pass the "dead points" during cycling phase $\left(0^{\circ}\right.$ and $180^{\circ}$, full extension/full flexion). Our pilot could not succeed to complete the pedaling cycle once fatigue appeared and sometimes from the beginning of training. Furthermore, the cocontraction balance between hamstrings and quadriceps muscle activations has a critical influence at these particular dead points and with time the equilibrium initially tuned is becoming less optimal (fatigue, movements on the seat...). The fixed-gear wheel was also supported by Szecsi 2007 et al. ${ }^{19}$ results, where the forces smoothing introduced by the use of a fixed-gear improved endurance. The gear ratio was set to be equal to 1.22 (number of rear wheel spins for one complete crank spin, or ratio between the chainring and the rear sprocket). Calculated with the 24 " rear wheel, we chose this ratio in order to best match between the optimal literature cadence, ${ }^{19}$ and the speed needed to reach 750 meters in 8 minutes, i.e. about $47 \mathrm{RPM}(5.6 \mathrm{~km} / \mathrm{h})$. The classical approach of FES-cycling is to predefine muscular activation patterns regarding the crank angle needed for the timing of the stimulation. The Berkelbike Pro® is equipped with such a crank sensor that can be connected to a stimulator commercialized by the company. A similar crank angle encoder (Baumer MDFK08) has been adapted on the crank axis of the FreeWheels trike in order to provide pedaling angle as an input to the stimulator. In addition, we have instrumented the device with different kind of sensors used to monitor the performances. Two speedometers were set, one with the screen oriented towards the pilot and a second one oriented to be visible to a person walking aside the trike. Finally, we added an ANT+ cardiac strap monitor to follow heart rate in response to effort.

\section{Stimulation Parameters}

During this study, we used the Berkelbike® FES-Box stimulator, an 8-channels battery powered stimulator. In cycling mode, a pre-programmed pattern based on crank angle, triggers the stimulation on and off cycles. Electrical stimulation was delivered on each leg via three stimulation channels composed each one of two platinum stimulating electrodes, size $4.5 \times 10 \mathrm{~cm}$, respectively located along the rectus femori/vastus lateralis muscles, the vastus medialis and the hamstrings (Fig. 3). A musculocutaneous flap was taken from the gluteus maximus as a treatment of a sacral pressure ulcer which prevented us to consider this muscle group as a candidate to electrical stimulation. Stimulation pattern was designed with individualized quadriceps. On each leg, the first channel dedicated to the rectus femori/vastus lateralis muscles was set to be effective during the pushing phase (full flexion to vertical crank), meanwhile the second channel was meant to later activate the vastus medialis, from the time where the 


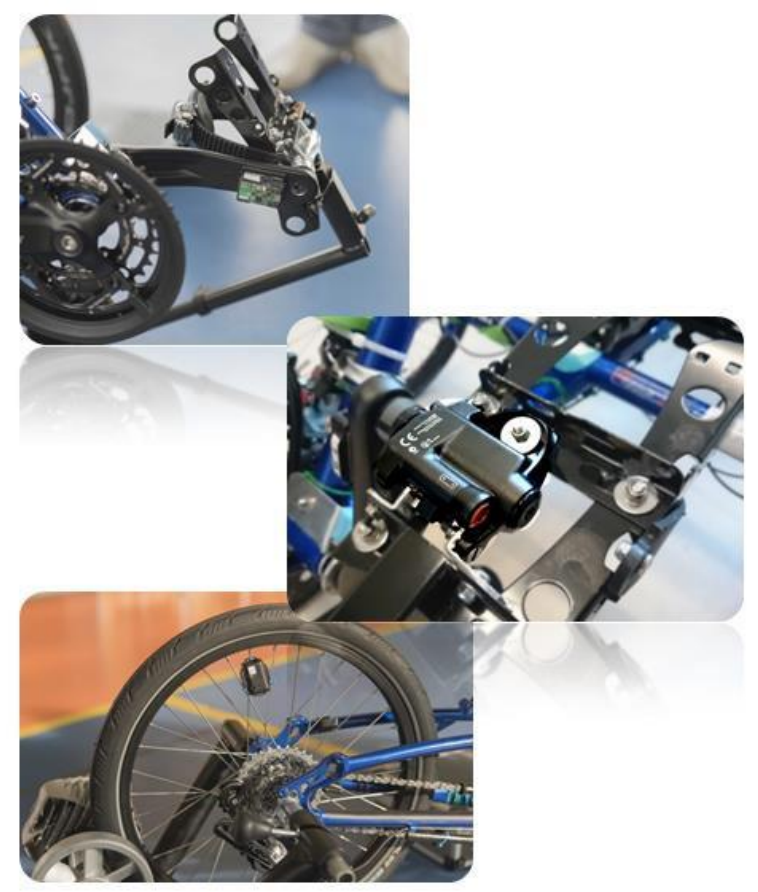

Fig 7. Power is computed via force pedals, cadence and speed are monitored via two inertial sensors (on the crank and rear wheel)

pedal was vertical to the full extension position. Hamstrings were respectively activated just before full extension and until the dead point was passed. Each training sessions were initiated with a warm-up phase, either set on a home trainer or rolling on a flat surface, before proceeding to the endurance race. During the warm-up phase, stimulation frequency was set to a lower level $(20 \mathrm{~Hz})$ than during the race $(30 \mathrm{~Hz})$. In both cases, we used a rectangular charge-balanced biphasic pulse stimulation with a pulsewidth of $400 \mu \mathrm{S}$ per phase, an interpulse of $200 \mu \mathrm{S}$ and a maximum intensity set to $150 \mathrm{~mA}$. The pilot controlled the stimulation intensity based on the speedometer information in order to fit the desired cadence of 47RPM. By pressing a button on the stimulator, he could gradually increase the level of stimulation on all the channels by $4.5 \mathrm{~mA}$ steps when the speed tended to go below $5.6 \mathrm{~km} / \mathrm{h}$.

\section{Training information}

A one year (12 mo.) training program was designed, divided into two stages:

1. from months M1 to M6: home based, two to three times a week, stimulation program of the lower limb muscles (quadriceps / hamstrings) during 30 minutes of isometric training using CEFAR $\AA$ Physio 4 stimulator and the following stimulation parameters: rectangular charge-balanced biphasic pulse stimulation with a pulsewidth of $300 \mu \mathrm{S}$ per phase and a ramp up, sent at $30 \mathrm{~Hz}$ during 10 s followed by $3 \mathrm{~s}$ of rest. At the start of each month, a mapping determined the intensity of stimulation needed to obtain contraction of the electrostimulated muscle and the intensity needed to reach a $4 / 5$ score on the MRC (Medical Research Council) scale.

2. from M7 to M11: FES-cycling training program, two to three times a week, on an instrumented trike. Either set on a home trainer or rolling outdoor and indoor on a flat surface. Stimulations parameters were modulated on the basis of progress.

From the beginning of the training, psychological and functional variables were also continuously monitored (self esteem, life quality, etc... ).

\section{Results}

\section{Performances}

FES-bike race was divided into two main steps, a qualification race and a final race. Our pilot was successfully qualified after a first race $(670 \mathrm{~m}$ in $430 \mathrm{~s}$, Fig.4) and could take part in the final race. The average speed during the final race was of $5.71 \mathrm{~km} / \mathrm{h}$ where he reached a maximal speed of $6.26 \mathrm{~km} / \mathrm{h}$. He finished $6 \mathrm{th}$ out of 12, accomplishing the whole race in $467 \mathrm{sec}$. Our cyclist reached his best performance one week before Cybathlon when he succeeded to achieve a distance of $1820 \mathrm{~m}$ on the ICE trike in stationary mode and $760 \mathrm{~m}$ rolling outdoor on a flat surface (Fig.5). Overcoming some technical issues, the functional performances dramatically and continuously increased from M11 (Fig. 5). Speed and endurance goals were successfully reached at M12 and kept evolving at M13 (1080m in 14min, Fig. 6).

\section{Feasibility}

The total cost of the device including the tricycle and the stimulator is estimated to $7650 €(4500 €$ for the stimulator, 3000 euros for the tricycle, and the rest for mechanical adaptations). The solution has the advantage to be easily duplicated. The choices made on its adaptation were driven by the Cybathlon objective: to cover $750 \mathrm{~m}$ in less than $8 \mathrm{~min}$. The system should be adapted and optimized to allow better performances based on the acquired knowledge. The stimulation patterns are designed through an individual manual tuning, with no general rule, making the procedure, at this stage, hardly feasible by an uninitiated user outside of a clinical environment

\section{Discussion}

Our participation into this human adventure was a team success. Despite numerous practical issues the initial goal was reached. We have not yet been able to objectivize and quantify all the technical choices and their consequences made to reach the Cybathlon goals. During the development phase we equipped the tricycle with inertial sensors (one on the crank and a second one on the rear wheel) and pedal power meters (Powertap P1, Fig. 7), using ANT+ wireless communication protocol and a specifically developed software. The 
original idea was to modulate stimulation parameters and patterns regarding pedaling cycle and produced forces. However, the feature which was supposed to give us a real time access to the stimulator parameters had not been ready on time before Cybathlon competition. Moreover, the pedal power meters were not sensitive enough to ensure an accurate measurement of the weak produced forces and to be usable as input to close loop control. Meanwhile, the different figures monitoring speed during training and races (Fig. 4 and Fig. 6) show the manual control of intensity level regarding cycling speed was really close to the initially automated considered one (consign of average speed set at $5.6 \mathrm{~km} / \mathrm{h}$, average recorded speed during final race of $5.71 \mathrm{~km} / \mathrm{h}$ ). The covering surface of the track also represented an important factor which drastically affected the obtained performances all along training and race sessions and could not have been quantified. The pilot training was part of a research protocol in which a longitudinal observation was achieved. The aim was to follow the evolution of performances as well as physiological and psychological parameters. Our pilot demonstrated a global acceptance of the participation into this 1 year protocol and an improvement along the experience of psychological variables such as life quality and self-esteem could be observed. Taking part in a competition as the final aim of the training program demonstrated a real benefit on training constraints tolerance. ${ }^{20-27}$ This experience and the interactions with other teams breaks new grounds to innovative and generic FES-cycling based training programs for an important range of SCI subjects. However, the constraints and the reality behind people with SCI daily lives are far from the Cybathlon FES bike context and require numerous improvements such as further investigations to enable a safe recreational use in complete autonomy of this activity.

\section{List of acronyms}

AIS - Abbreviated Injury Scale.

CAMIN - Control of Artificial Movement \& Intuitive Neuroprosthesis

COS - Centre d'Orientation Sociale

CPP - Comité de Protection des Personnes

DXA - Dual Energy X-Ray Absorptiometry

FES - Functional Electrical Stimulation

ICE - Inspired-Cycle-Engineering

INRIA - Institut National de Recherche en Informatique et Automatique

MRC - Medical Research Council

RCB - Recherche et Collection Biologique

RPM - Revolutions Per Minute

SCI - Spinal Cord Injuries

\section{Author's contributions}

BS is the principle author and responsible for the technical integration and software design of the FreeWheels project. CF and AD were responsible for the clinical protocol, pilot training and follow up. CA was the leader of the team. All the authors read and approved the final version of the manuscript.

\section{Acknowledgments}

This research was supported by a Neuroglia foundation for neurosciences grant and an INRIA internal financial support. Part of this work has been done in collaboration with EMA team (University of Brasilia) with the help of CACAO associate team (INRIA)

\section{Conflict of Interest}

The authors declare no potential conflict of interests.

\section{Ethical Publication Statement}

We confirm that we have read the Journal's position on issues involved in ethical publication and affirm that this report is consistent with those guidelines.

\section{Corresponding Author}

Benoît Sijobert, INRIA - LIRMM Université de

Montpellier, Montpellier, France.

E-mail: benoit.sijobert@inria.fr

E-mails of co-authors

Christine Azevedo: christine.azevedo@inria.fr

Charles Fattal: cfattal@lachataigneraie.fr

Anne Daubigney: anne.daubigney@gmail.com

\section{References}

1. FREEwheels official website. Available from: http://freewheels.inria.fr.

2. Zhang Q, Hayashibe M, Azevedo-Coste C. Evoked electromyography-based closed-loop torque control in functional electrical stimulation. IEEE Trans Biomed Eng 2013;60:2299-307.

3. Guiraud D, Azevedo Coste C, Benoussaad M, Fattal C. Implanted functional electrical stimulation: case report of a paraplegic patient with complete SCI after 9 years. J Neuroeng Rehabil 2014;11:15. Available from: http://jneuroengrehab.biomedcentral.com/articles/1 0.1186/1743-0003-11-15

4. Azevedo Coste C, Jovic J, Pissard-Gibollet R, Froger J. Continuous gait cycle index estimation for electrical stimulation assisted foot drop correction. J Neuroeng Rehabil 2014;11(1):118.

5. Bó APL, Azevedo-Coste C, Geny C, etal. On the Use of Fixed-Intensity Functional Electrical Stimulation for Attenuating Essential Tremor. Artif Organs 2014;38:984-91.

6. Sijobert B, Azevedo C, Andreu D, et al. Effects of sensitive electrical stimulation-based somatosensory cueing in Parkinson's disease gait and Freezing of Gait assessment. Artif Organs 2017, 41: E222-E232. doi:10.1111/aor.13059.

7. Glaser RM. Physiologic aspects of spinal cord injury and functional neuromuscular stimulation. Cent Nerv Syst Trauma 1986;3:49-62. 
8. Fornusek C, Davis GM. Cardiovascular and Metabolic Responses During Functional Electric Stimulation Cycling at Different Cadences. Arch Phys Med Rehabil 2008;89:719-25.

9. Berkelmans R. Fes cycling. J Autom Control 2008;18:73-6.

10. Newham DJ, Donaldson NDN. FES cycling. Acta Neurochir Suppl 2007;97 PART 1:395-402.

11. Wilder RP, Jones E V, Wind TC, Edlich RF. Functional electrical stimulation cycle ergometer exercise for spinal cord injured patients. J Long Term Eff Med Implants 2002;12:161-74.

12. Guimarães JA, da Fonseca LO, Dos Santos-CoutoPaz CC, et al. Towards Parameters and Protocols to Recommend FES-Cycling in Cases of Paraplegia: A Preliminary Report. Eur J Transl Myol 2016;26:6085.

13. Coste CA, Mayr W, Bijak M, et al. FES in Europe and Beyond: Current Translational Research. Eur J Transl Myol 2016;26(4):6369.

14. Figoni SF, Glaser RM. Acute hemodynamic responses of spinal cord injured individuals to functional neuromuscular stimulation-induced knee extension exercise. J Rehabil Res Dev 1991;28:9-18.

15. PL J, MS N. Modes, benefits, and risks of voluntary electrically induced exercise in persons with spinal cord injury. J Spinal Cord Med 2001;24:10-8.

16. Peri E, Ambrosini E, Pedrocchi A, et al. Can FESAugmented Active Cycling Training Improve Locomotion in Post-Acute Elderly Stroke Patients? Eur J Transl Myol 2016;26:6063.

17. Eser PC, Donaldson N de N, Knecht H, Stüssi E. Influence of different stimulation frequencies on power output and fatigue during FES-cycling in recently injured SCI people. IEEE Trans Neural Syst Rehabil Eng 2003;11:236-40.

18. Pollack SF, Axen K, Spielholz N, et al. Aerobic training effects of electrically induced lower extremity exercises in spinal cord injured people. Arch Phys Med Rehabil 1989;70:214-9.
19. Szecsi J, Krause P, Krafczyk S, et al. Functional output improvement in FES cycling by means of forced smooth pedaling. Med Sci Sports Exerc 2007;39:764-80.

20. Azevedo Coste C, Bergeron V, Berkelman R, et al. Comparison of strategies and performance of functional electrical stimulation cycling in spinal cord injury pilots for competition in the first ever Cybathlon. Eur J Transl Myol 2017;27:251-4.

21. Berkelmans R, Woods B. Strategies and performances of Functional Electrical Stimulation Cycling using the BerkelBike with Spinal Cord Injury in a competition context (CYBATHLON). Eur J Transl Myol 2017;27:255-8.

22. Laubacher M, Aksöz EA, Bersch I, Hunt KJ. The road to Cybathlon 2016 - Functional electrical stimulation cycling Team IRPT/SPZ. Eur J Transl Myol 2017;27:259-64.

23. Guimarães JA, Oliveira da Fonseca L, de Sousa AC, et al. FES Bike Race preparation to Cybathlon 2016 by EMA team: a short case report. Eur J Transl Myol 2017;27:272-78.

24. Metani A, Popovic-Maneski L, Mateo S, et al. Functional electrical stimulation cycling strategies tested during preparation for the First Cybathlon Competition - a practical report from team ENS de Lyon. Eur J Transl Myol 2017;27:279-88.

25. McDaniel J, Lombardo LM, Foglyano KM, et al. Cycle Training Using Implanted Neural Prostheses: Team Cleveland. Eur J Transl Myol 2017;27:289-94.

26. Arnin J, Yamsa-ard T, Triponyuwasin P, Wongsawat Y. Development of practical functional electrical stimulation cycling systems based on an electromyography study of the Cybathlon 2016. Eur J Transl Myol 2017;27:295301.

27. Leung WC, Tong RKY, Wang X, et al. The Effectiveness of Functional Electrical Stimulation (FES) in On-Off Mode for enhancing the cycling performance of Team Phoenix at 2016 Cybathlon. Eur J Transl Myo 2017;27:302-6. 